\title{
Recent Advances in Flower Pollination Algorithm
}

\author{
Nabil Diab \\ Department of Computer Science, \\ Faculty of Computers and Informatics, \\ Higher Technology institute $10^{\text {th }}$ of Ramadan, \\ Egypt.
}

\author{
Emad El-Sharkawy \\ Faculty of Information Systems and Computer \\ Science, October 6 University \\ Egypt.
}

\begin{abstract}
Flower Pollination Algorithm (FPA) is a nature inspired algorithm based on pollination process of plants. Recently, FPA has become a popular algorithm in the evolutionary computation field due to its superiority to many other algorithms. As a consequence, in this paper, FPA, its improvements, its hybridization and applications in many fields, such as operations research, engineering and computer science, are discussed and analyzed. Based on its applications in the field of optimization it was seemed that this algorithm has a better convergence speed compared to other algorithms. The survey investigates the difference between FPA versions as well as its applications. To add to this, several future improvements are suggested.
\end{abstract}

Keywords: flower pollination algorithm; inspired algorithms; optimization; hybridization

\section{INTRODUCTION}

Mathematical model for system modeling and objective function is used in most of the optimization algorithms but establishing mathematical model is not efficient due to its high cost of solution time, classical optimization algorithms are not efficient due to many causes such as ineffectiveness in adaptation of a solution algorithm, the dependency on type of objective and constraints, on type of variables used in modeling of problem, on solution space (convex, nonconvex), number of decision variable, and the number of constraints in problem modeling that's why classical optimization algorithms are insufficient in large-scale combinational and non-linear problems. Most of algorithms solve models which have a certain type of object function or constraints. However, optimization problems in many different areas such as computer science, management, and engineering require concurrently different types of decision variables, object function, and constraints in their formulation. Therefore, meta-heuristic optimization algorithms become quite popular methods in recent years, because they provide good computing power and easy conversion, and due to its flexibility to convert meta-heuristic program from a single objective function problem to a multi objective problem or a different problem.

Recently, Yang [1] developed a new Flower pollination algorithm that draws its inspiration from the flow pollination process of flowering plants. FPA testing results in many fields proved their ability to be used in a wide range of optimization problems and also their ability to provide better performance in comparison with other traditional optimization techniques. This paper introduces improvements, hybridization and applications of FPA.

This paper is organized as follows: after introduction, the original Flower pollination algorithm is briefly introduced. In section 3, Multi-objective FPA is described. Section 4 the Binary FPA version is introduced. The algorithm improvements are taken in section 5, while the real-world applications of FPA are discussed in section 6. Finally, conclusions and suggestions for future work are presented in section 7.

\section{FLOWER POLLINATION ALGORITHM}

FPA, inspired by the flow pollination process of flowery plants, was developed in 2012 by Xin-She-Yang [1]. The following 4-rules are used as a matter of convenience.

1.Biotic and cross-pollination is considered as global pollination process with pollen-carrying pollinators performing L'evy flights.

2. Abiotic and self-pollination are considered as local pollination.

3. Flower constancy can be considered as the reproduction probability is proportional to the similarity of two flowers involved.

4. Local pollination and global pollination is controlled by a switch probability $\mathrm{p} \in[0,1]$.

In order to formulate updating formulas, we have to convert the aforementioned rules into updating equations. For example, in the global pollination step, flower pollen gametes are carried by pollinators such as insects, and pollen can travel over a long distance because insects can often fly and move in a much longer range[1].Therefore, Rule 1 and flower constancy can be represented mathematically as:

$$
x_{i}^{t+1}=x_{i}^{t}+\gamma L(\lambda)\left(x_{i}^{t}-B\right)
$$

Where $x_{i}^{t}$ is the pollen $\mathrm{i}$ or solution vector xi at iteration $\mathrm{t}$, and $\mathrm{B}$ is the current best solution found among all solutions at the current generation/iteration. Here $\gamma$ is a scaling factor to control the step size. In addition, $L(\lambda)$ is the parameter that corresponds to the strength of the pollination, which essentially is also the step size. Since insects may move over a long distance with various distance steps, we can use a Le'vy flight to imitate this characteristic efficiently. That is, we draw $\mathrm{L}>0$ from a Levy distribution:

$$
L \sim \frac{\lambda \Gamma(\lambda) \sin (\pi \lambda / 2)}{\pi} \frac{1}{S^{1+\lambda}},\left(S>>S_{0}>0\right)
$$

Here, $\Gamma(\lambda)$ is the standard gamma function, and this distribution is valid for large steps $s>0$.

Then, to model the local pollination, both Rule 2 and Rule 3 can be represented as:

$$
x_{i}^{t+1}=x_{i}^{t}+U\left(x_{j}^{t}-x_{k}^{t}\right)
$$


Where $x_{j}^{t}$ and $x_{k}^{t}$ are pollen from different flowers of the same plant species. This essentially imitates the flower constancy in a limited neighborhood. Mathematically, if $x_{j}^{t}$ and $x_{k}^{t}$ comes from the same species or selected from the same population, this equivalently becomes a local random walk if we draw $U$ from a uniform distribution in $[0$, 1].Though Flower pollination activities can occur at all scales, both local and global, adjacent flower patches or flowers in the not-so-far-away neighborhood are more likely to be pollinated by local flower pollen than those faraway. In order to imitate this, we can effectively use the switch probability like in Rule 4 or the proximity probability $\mathrm{p}$ to switch between common global pollination to intensive local pollination. To begin with, we can use a naive value of $p=0.5$ as an initially value. A preliminary parametric showed that $p=0.8$ might work better for most application. The pseudo code of FPA is shown in Figure 1.

\section{Flower Pollination Algorithm}

Objective function min or max $f(x), x=(x 1, x 2, \ldots, x d)$

Initialize a population of $n$ flower/pollen gametes with random solutions.

Find the best solution in the population

Define a switch probability

Define a stopping criterion

While ()

For $i=1: n$ (all $n$ flowers in the population)

If rand $<p$,

Draw a (d-dimensional) step vector $L$ which obeys Levy distribution

Do Global pollination via

Else

Draw from a uniform distribution in [0,1]

Do Local pollination via

End If

Evaluate new solutions

If new solutions are better, than update them in the population

Find current best solution

End While

Fig.1: Pseudo code of standard Flower Pollination Algorithm (FPA).

To simplify the implementation of this algorithm it is assumed that each flower produces only one flower, There are some applications that require to assign each flower with multiple pollen gametes and each plant with multiple flowers such as Graph coloring, image compression and multiobjective optimization problems.

\section{FPA MULTI-OBJECTIVE VERSION}

\section{[3]}

Multi-objective optimization problems are typically complex problems. The methods for solving multi-objective problems differ from algorithms for single objective optimization.

For single objective optimization, the optimal solution can often be a single point in the solution space, while for biobjective optimization, the Pareto front forms a curve, and for tri-objective cases, it becomes a surface. In fact, higher dimensional problems can have extremely complex hypersurface as its Pareto front ([4];[5];[6];[7]) Consequently, it is typically more challenging to solve such high-dimensional problems. Real-world design problems in engineering and industry is considered as multi-objective optimization that requires to produce many points on the Pareto front for good approximations. In order to compare the performance of the proposed MOFPA with other multi-objective algorithms, we have selected a few algorithms with available results from the literature, such as vector evaluated genetic algorithm (VEGA) [8],NSGAII[9],multi-objective differential evolution (MODE)([10];[11]), differential evolution for multi-objective optimization(DEMO)[12],multi-objective bees algorithms[13], and strength Pareto evolutionary algorithm(SPEA) [14].

The experimental results for 11 test functions and two design examples suggest that MOFPA is a very efficient algorithm for multi-objective optimization. The algorithm can deal with highly nonlinear, multi-objective optimization problems with complex constraints and diverse Pareto optimal sets.

\section{FPA BINARY VERSION}

Binary-constrained version of the Flower Pollination Algorithm [15] (FPA) is implemented for feature selection, BFPA determines the features that compose the final set by using Boolean search space, this method was applied to some public and private datasets. Numerical experiments in comparison with Particle Swarm Optimization, Harmony Search and Firefly Algorithm have demonstrated the suitability of the FPA for feature selection.

\section{IMPROVEMENTS}

There are a lot of significant research studies that aim to improve the FPA performance, by algorithm modification or effective hybridization with other Nature Inspired Algorithms. The improved algorithms support researchers to solve more optimization problems.

\subsection{Modification}

\subsubsection{Modified flower pollination algorithm (MFPA) [16]}

After testing the FPA they found an important impact on the computational burden and convergence solution in the initial population and switching from local to global pollination.

The following modifications improve the algorithm performance:

A. Looking for the Best Initial Condition

B. Switching the Local to Global Pollination Process

This modification eliminates the use of the probability switch and combines Lévy flights with Brownian motion into a single random walk equation. MFPA has been successfully implemented to solve optimal power flow problem. The algorithm has been tested on the IEEE 30-bus system. The experimental results show results enhancement compare to other meta-heuristics algorithms. The main advantage of the MFPA is to find fitter initial solutions with improvement of the switching process. Both modifications let the algorithm reach the convergence with minimum number of iterations and little processing time. 


\subsubsection{Improved Flower Pollination Algorithm with Chaos [17]}

The definite integrals are useful in a wide range of applications in many regions such as operation research, computer science, mathematics, mechanics, physics, and engineering. Improved Flower pollination algorithm with chaos (IFPCH) introduced by [17] for solving definite integrals. Numerical results show that the algorithm offers an effective way to calculate numerical value of definite integrals, it can converge to the best solution and it has a high convergence rate, high accuracy and robustness.

\subsection{Hybridization}

After making several hybridization between the flower pollination algorithm and other inspired meta-heuristic algorithms, there is certainty that Hybridization improve the performance of the FPA to reach the optimal solution faster than the algorithm itself in a short period of time and to reduce the algorithm limitations.

\subsubsection{Flower pollination algorithm with Chaotic Harmony Search (FPCHS) [18]}

Integration of flower pollination algorithm with chaotic harmony search aims to improve the searching accuracy, the planned algorithm is used to solve Sudoku puzzles. The algorithm has been tested on a set of Sudoku problems. The results verify that the planned algorithm is more efficient compared with other algorithms.

\subsubsection{Flower pollination algorithm with genetic algorithm (FPA-GA) [19]}

In the FPA-GA the flower pollination algorithm (FPA) and genetic algorithm (GA) are integrated to solve constrained optimization problems. The experimental results of testing the FPA-GA in seven benchmark optimization problems verify that the proposed algorithm is very effective and can be extended to solve other problem such as combinatorial optimization problems.

\subsubsection{Flower pollination algorithm with particle swarm optimization (FPPSO) [20]}

Integration of Flower pollination algorithm with particle swarm optimization aims to improve the searching accuracy, the hybrid algorithm is used to solve constrained optimization problems, FPPOS has been validated using several benchmark mathematical and engineering design problems and the results prove that the FPPOS is more efficient at finding global optimal solution than other algorithms.

\subsubsection{Flower pollination algorithm with differential evolution optimization algorithm (DE-FPA) [21]}

New hybrid population based algorithm which has of the exploration capability of differential evolution and also inherits the directed movement towards the global best and local search from the flower pollination algorithm this proposed algorithm was handling the drawbacks of the individual algorithms such as slow convergence to the global optima at the differential evolution .DE-FPA has been tested with some benchmark test functions, the results clearly establish that the DE-FPA have the superiority over both the $\mathrm{DE}$ and FPA in terms of performance and convergence rate. And a novel concept of dynamic adaptive weight is introduced for faster convergence than the individual algorithms.

\subsubsection{Flower pollination algorithm with $\mathrm{K}$ - Means algorithm (FPAKM) [22]}

FPAKM is a hybrid data clustering approach using Flower Pollination Algorithm and K-Means, K-Means algorithm solve the data clustering problems in a very fast way but find the local optimum solution, the flower pollination algorithm is the global optimization technique that eliminate this drawback, FPAKM has been tested on eight datasets, The experimental results prove that FPAKM find optimal cluster centers and that is better than these individual algorithms.

\subsubsection{Flower pollination algorithm, differential evolution algorithm (HFPA) with time varying fuzzy selection mechanism (HFPA-TVFSM)} [23]

In the HFPA the flower pollination algorithm (FPA) and differential evolution (DE) algorithm are integrated to solve wind-thermal dynamic multi-objective optimal dispatch problem that improves the exploration and exploitation potential of the flower population which is conducting the search, in this problem there are more constraint such as minimization of cost, emission and losses, and other complex constraints like valve point loadings, ramp limits, prohibited zones and spinning reserve. To solving multi-objective problems HFPA is integrated with a 5-class, 3-step time varying fuzzy selection mechanism (TVFSM) that effectively searches the best compromise solution (BCS) .HFPA-TVFSM is tested and validated on two wind-thermal test systems from literature, the results prove that the hybrid algorithm performs efficiently and all constraints are satisfied.

\subsubsection{Flower pollination algorithm by Artificial Bees (FPAB) and Biogeography Based Optimization Algorithm (FPAB/BBO) [24]}

Remote sensing has been used for the classification of satellite image on a very large scale. A hybridization of Flower Pollination by Artificial Bees (FPAB) and Biogeography Based Optimization Algorithm has been used to classify a satellite image as an efficient land cover classifier for satellite image, this resulted in highly accurate classification of the satellite image is obtained by using FPAB/BBO and many of the land cover features are identified much more clearly

\subsubsection{Flower pollination algorithm with Clonal Selection Algorithm (MFPA) [25]}

The Flower Pollination Algorithm is hybridized with the Clonal Selection Algorithm, The Experimental results of testing the Algorithm in 23 optimization benchmark problems verify the algorithm superiority compared to other famous optimization algorithm such as Simulated Annealing, Genetic Algorithm, Flower Pollination Algorithm, Bat Algorithm, and 
Firefly Algorithm, and also verify that the algorithm is able to find more accurate solutions than the other optimization techniques.

\subsubsection{Flower Pollination Algorithm with Simulated Annealing Algorithm (FPSA) [26]}

The standard flower pollination algorithm (FPA) with simulated annealing are integrated to enhance the search performance and speeds up the global convergence rate, FPA generate initial state of simulated annealing and new solutions , FPSA has been tested in structural engineering optimization problems. The experimental results prove that this method is accurate in finding the best solution and have fast convergence speed compared to existing algorithms.

\subsubsection{Flower Pollination Algorithm with Tabu Search Algorithm (TS-FPA) [27]}

Integration of flower pollination algorithm (FPA) and Tabu Search Algorithm to solve unconstrained optimization problems, these technique eliminate the limitations of FPA in population diversity and convergence precision. TS-FPA is validated by ten benchmark functions. The experimental results show the algorithm high stability and it also has a fast convergence speed.

\section{APPLICATIONS}

FPA is validated in several applications and all the experimental results are more efficient compared to other algorithms ,Furthermore FPA finds global optimal solution in a short period of time. In recent years ,FPA has been applied by several researchers to various optimization problems in computer science, operations research, engineering and science among which we mention the important applications.

\subsection{Computer Science}

Computer science is the scientific and practical approach to computation, computer science aims to identifying certain computer science concepts that can be used directly in solving real world problems. Meta-heuristic algorithms are a higher level procedure or heuristic designed to find, generate, or select a heuristic. Metaheuristic algorithms applied in many CS applications provide a sufficiently good solution to optimization problems.

FPA is a nature inspired metaheuristic algorithm implemented in CS applications to find optimal solution .The numerical results clearly show that the FPA gives better results that prove the algorithm suitability for solving different real world problems .

Some applications of FPA in Computer science field:

\subsubsection{Robust and Efficient 'RGB' based Fractal Image Compression [28]}

Pollination Based Optimization (PBO) is a new technique based on Pollination Based Optimization which improve the fractal image compression FIC ,these technique used to classify the phantom, satellite and rural image dataset the experimental results show that $\mathrm{PBO}$ performance is better than other optimization technique, using PBO reduce the encoding time and improves the visual quality of image in comparison with FCI.PBO results encouraged researchers to use these technique many area of the multimedia system's world.

\subsubsection{Wireless Sensor Network Lifetime Global Optimization [29]}

To achieve the global optimization for WSN lifetime using flower pollination algorithm rather than the classical LEACH algorithm .FPA selects the best $\mathrm{CHs}$ distribution that guarantees a routing optimization with the minimum communication links` cost between nodes within each cluster. This also optimize the sum of nodes energy (WSN total energy) based on the fixed number of cluster heads that minimize the overhead of data aggregation and the management and control signals for each cluster head. Results show an enhancement in the wireless sensor network lifetime ,network stability and overall network lifetime.

\subsubsection{Retinal vessel segmentation [30]}

FPA is implemented to search for the optimal clustering of the given retinal image into compact clusters under some constrains , at local search period. Shape features are used to enhance the clustering results, this approach is tested on a publicly available databases drive of retinal images. Results prove that performance enhancement compared to other art techniques.

\subsubsection{Graph coloring problem [31]}

Flower pollination algorithm is a recent nature inspired algorithm for continuous optimization, a discrete version of the algorithm was used to solve graph coloring problem. The performance of the algorithm is evaluated on benchmark instances set, the experimental results ensure it achieves the exact solution in almost cases.

\subsubsection{Planar Graph Coloring Problem [31,32]}

Local greedy flower pollination algorithm (LGFPA) was implemented for solving planar graph coloring problem based on local swap strategy, local reverse strategy. These algorithm can solve the planar graph coloring problem using four-colors more efficiently and accurately. The experimental results show that this algorithm can get smaller average iterations than basic flower pollination algorithm (FPA), particle swarm optimization (PSO), differential evolution (DE), and can obtain higher successful coloring rate.

\subsection{Operation research}

Operation research is often considered to be a sub-field of mathematic aim to find optimal or near-optimal solutions to complex decision-making problems by using optimization techniques .Many meta-heuristic optimization methods have been developed to solve such difficult optimization problems. FPA is a nature inspired metaheuristic algorithm implemented in OR applications to solve optimization problems.

The numerical results clearly show that the FPA gives better results that prove the algorithm suitability for solving different difficult optimization problems.

Some applications of FPA in Operation research field: 


\subsubsection{Integer Programming Problems [33]}

Integration of the flower pollination algorithm with chaos theory (IFPCH) to solve integer programming problems, the new solution produce by FPA taken by the IFPCH that rounded the parameter values to the closest integer .This approach has been tested by several problems, the experimental results prove the superiority of the new approach over the standard FPA in terms of efficiency and success rate.

\subsubsection{Large Integer Programming Problems $[33,34]$}

An improved version of Flower pollination Meta-heuristic Algorithm, (FPPSO) that combines the standard flower pollination algorithm (FPA) with the particle swarm optimization (PSO) algorithm to improve the searching accuracy, using this algorithm to solving integer programming problems result in obtain the optimal solution within less computation.

\subsubsection{Ratios Optimization Problems [35]}

Integration of the Flower Pollination Algorithm with Chaos Theory (IFPCH) to solve large-scale ROP with an optimal solution at a finite point and an unbounded constraint set.The technique is tested using several ROP benchmark. The test aims to prove the capability of the IFPCH to solve any type of ROPs. The experimental results show the feasibility, effectiveness, and robustness of the technique. The results revealed the superiority of the technique among others in computational time.

\subsubsection{Dimension by Dimension Improvement [36]}

FPA has been work with three optimization strategies (local neighborhood search strategy, dimension by dimension evaluation and improvement strategy, and dynamic switching probability strategy) to improve FPA deficiencies . The experimental results by applied 12 typical standard benchmark functions simulation show that DDIFPA algorithm has strong global searching ability and local optimization ability, also can improve the convergence speed and the quality of solutions effectively.

\section{.6.2.5 Optimal Unmanned Undersea Vehicle Path Planning Problem [37]}

Implementation of flower pollination algorithm to solve the UUV path planning problem in $2 \mathrm{D}$ and $3 \mathrm{D}$ space. Optimization strategies of particle swarm optimization are applied to the local search process of IFPA to enhance its search ability, and the solution exposed to dimension by dimension based update and evaluation strategy. This approach can accelerate the global convergence speed. The experiment results prove that this approach is more effective and feasible in UUV path planning in $2 \mathrm{D}$ and $3 \mathrm{D}$ space compared to nine population based algorithms.

\subsubsection{Selection of University academic credits} [38]
Implementation of FPA to make optimal selection of an elective in Bachelor level and to interact with the different values associated with the achievement of the term loans and the cost-benefit for every student in a minority group and comparing their choices of electives with respect to the group. The algorithm is tested in case of four scholar minorities studies in a University with approximately 87 educational on Bachelor level.

\subsection{Electrical Engineering}

In electric power market, it is too difficult to offer electric power to customer with high quality and least cost because of several complicated problems. There is a need to find optimal solution for operation and design of an efficient power system. To find optimal solution FPA is implemented for different problems. The numerical results clearly show that the FPA gives better results that prove the algorithm suitability for different power system optimization problems. Some applications of FPA in electrical field:

\subsubsection{Optimal Reactive Power Dispatch Problem [39]}

Optimal Reactive Power Dispatch Problem ORPD objective is reduce losses and stability of the system improvement. FPA is implemented in the standard IEEE-30 bus system and results are compared to other recently reported work and it is clear that this algorithm performs in a better way.

\subsubsection{Power Loss Reduction on Radial Distribution System problem [40]}

FPA implemented to decides the locations and size of capacitors to realize the optimum sizable reduction in active power loss and significant improvement in voltage profile. This method is tested on 10, 15, 69 and85-bus radial distribution systems, the results found is better than the other methods in terms of the quality of solution.

\subsubsection{Optimal Line Flow in Conventional Power System problem [41]}

Euclidean affine flower pollination algorithm (EFPA) used to addresses the optimal line flow OLF constraint for minimizing the fuel cost, loss, emission and voltage stability index for all generators in distributed power systems ,EFPA is performed on IEEE 30 bus system and IEEE 57 bus system, Results proved that the EFPA optimization is efficient for OLF constraint.

\subsubsection{Linear antenna array optimization problem [42]}

Apply FPA to the electromagnetic and antenna community for the optimization of linear antenna arrays. FPA was applied to obtain optimized antenna positions in order to achieve desired array pattern with minimum SLL along with null placement in specified directions. Results have been compared to conventional array (non-optimized), and with arrays optimized using other nature-inspired evolutionary algorithms such as ACO, CSO and PSO, Consequent FPA outperforms the other evolutionary algorithms and at times it yields a similar performance. 


\subsubsection{Optimal Control in Multi-Machine System with GUPFC Problem [43]}

Generalized unified power flow controller (GUPFC) problem is to tuning the control parameters of PSS and POD, Flower pollination algorithm implemented in multi-machine system to provide parameters of two stage lead-lag controller .The results demonstrate that Flower pollination algorithm can be used as a method of tuning the control parameters of PSS and POD in a multi-machine power system.

\subsubsection{Synthesis of Circular Array Antenna for Side lobe Level and Aperture Size Control [44]}

FPA is implemented for circular array synthesis to solve Side lobe level suppression, FPA adapts one and two degrees of freedom, namely, amplitude only and amplitude spacing. The experimental results prove the effectiveness of FPA compared to genetic algorithm (GA) and uniform circular array antenna (UCAA)

\subsubsection{Improvement of Voltage Profile using Shunt Capacitor in Radial Distribution System [45]}

A method of reducing the loss by placing shunt capacitors at the optimal location, using Loss Sensitivity Factor and FPA to determine capacitor placement make significant decrease in power loss, increase in voltage profile and decrease in total annual cost.

\subsubsection{Voltage Profile Improvement using Distributed Generation in 33-bus and 69-bus RDS [46]}

A method of reducing the loss by placing distribution generation at the optimal location, using Loss Sensitivity Factor and FPA to determine optimal allocation of distribution generation in a radial distribution system make significant decrease in power loss, increase in voltage profile ,this method is tested on 33-bus and 69-bus system, yields good results.

\subsubsection{Optimal sizing and locations of capacitors in radial distribution systems [47]}

Implementation of Flower Pollination Algorithm to allocate and determine capacitors sizes in various distribution systems. FPA is tested on 15, 69 and 118-bus radial distribution systems, results is better than the other algorithms such as Genetic Algorithm (GA), Particle Swarm Optimization (PSO),Plant Growth Simulation Algorithm (PGSA), Direct Search Algorithm (DSA), Teaching Learning-Based Optimization(TLBO), Cuckoo Search Algorithm (CSA), Artificial Bee Colony (ABC) and Harmony Search. FPA enhance the voltage profile, minimize the losses and total cost for various distribution systems.

\subsubsection{0 optimal placement and sizing of distributed generation in Distribution systems [48]}

A method of reducing the loss by placing Distributed generator at the optimal location and determine the optimal
DG size, using the index vector method and FPA to determine optimal allocation of Distributed generator in a radial distribution system and determine the optimal DG size make significant decrease in power loss, increase in voltage profile, this method is tested on 15-bus 34-bus and 69-bus system, yields good results.

\subsubsection{Distributed generations planning [49]}

A method of reducing the loss by placing Distributed generator at the optimal location and determine the optimal DG size, using the index vector method and FPA to determine optimal allocation of Distributed generator in a radial distribution system and determine the optimal DG size make significant decrease in power loss, increase in voltage profile, this method is tested on 33-bus 69-bus and 136-bus system, yields good results. Furthermore, the results obtained by the FPA algorithm are compared with other metaheuristic optimization techniques such as backtracking search optimization algorithm, artificial bee colony, and selection algorithm ,The outcomes verify that the FPA algorithm is efficient.

\subsubsection{Load Frequency Control for a Hydro- Thermal Deregulated Power System [50]}

FPA is implemented to tune the PI controller of the LFC to improve the dynamic response, by minimizing the Integral Square Error of the system. The results show that the FPA tuned PI controller improves the dynamic response of the deregulated system faster than the PI controller for different cases.

\subsubsection{Economic and emission dispatch problems [51]}

Implementation of Flower Pollination Algorithm (FPA) to solve ELD and CEED problems in power systems, Results prove that FPA have the superiority over other optimization algorithms for large scale power system with valve point effect in terms of total cost and computational time.

\subsubsection{Different Economic Load Dispatch Problems [52]}

Flower pollination algorithm is implemented to adjust real power generations for minimizing the fuel cost, FPA is tested on the standard IEEE-30 bus system and the results are compared with those of the other algorithms, The results are found improved and encouraging.

\subsubsection{Short Term Hydrothermal Scheduling} [53]

Implementation of improved flower pollination algorithm (IFPA) for solution of short term hydrothermal scheduling problem. In IFPA the local pollination process of FPA is controlled by adding a scaling factor and an additional intensive exploitation phase is added to tune and improve the best solution. The improved algorithm and FPA are tested by three different test cases .the experiment result provide the superiority of IFPA. 


\subsection{Other Fields}

Applications of FPA in other fields;

\subsubsection{Estimation of the Transition Matrix in Markov Chain Model of Customer Lifetime Value [54]}

FPA is implemented early in application about real health insurance data and it give efficient results in solving inverse problem in Markov Chain Model. Consequently FPA is applied to solve inverse problem of Customer Lifetime Value (CLV). For the study case, health insurance data is taken along with some arbitrary constant interest rates for the next five years.

\subsubsection{Application of the Flower Pollination Algorithm in Structural Engineering [55]}

\begin{abstract}
Structural engineering problems have various design constraints concerning structural security measures and practicability in production. Thus, optimization becomes an important part of the design process. FPA was implemented to solve structural engineering problems. The engineering problems are about pin-jointed plane frames, truss systems, deflection minimization of I-beams, tubular columns, and cantilever beams. The experimental results show that FPA is effective to find the best optimum results when compared to other method.
\end{abstract}

\section{CONCLUSION AND FUTURE WORK}

How to solve real world optimization problems is a question asked every day and researchers seek to find the answer ,therefore implementing Nature inspired algorithm has being one of the effective methods to solve these problems, since 2012 the Flower Pollination Algorithm proposed by Xin-She Yang [1] was implemented in many fields such as operation researches ,computer science and electrical engineering. This paper is a review which explores the FPA efficiency whether by implementation or by hybridization with other algorithms, the studies prove that FPA is a powerful tool in solving several optimization problems, a consequence FPA is one of the more commonly used algorithms until now. The future work will be focused on:

i. Applying binary flower pollination version to solve most popular constrained engineering optimization problems. ii. Applying binary flower pollination version to solve combinatorial optimization problem such as (Quadratic assignment problem, Traveling salesman problem, Graph Coloring problem, Knapsack problem, etc.).

iii. Applying FPA with fuzzy logic.

iv. Combining FPA with Neutrosophic logic.

v. Applying FPA to solve geographical information system applications

vi. Applying FPA to solve machine learning problems. vii. Proposing new adaptive mechanism to update FPA parameters.

viii. Analyzing the effect of hybridizing FPA with other EAs.

\section{REFERENCES}

[1] Xin-She Yang, "Flower pollination algorithm for global optimization", in: Unconventional Computation and Natural Computation 2012, Lecture Notes in Computer Science, Vol. 7445, pp. 240-249 (2012).

[2] Wikipedia article on pollination, http://en.wikipedia.org/wiki/Pollination.

[3] X. S. Yang, M. Karamanoglu, X. S. He," Flower Pollination Algorithm: A

Novel Approach for Multiobjective Optimization" in: Engineering Optimization, vol. 46, Issue 9, pp.1222-1237 (2014).

[4] Madavan, N. K., 2002. “ Multiobjective optimization using a pareto differential evolution approach.” in: Congress on Evolutionary Computation (CEC'2002), Vol. 2, NewJersey, IEEE Service Center, pp. 1145\{1150\}

[5] Marler, R. T. and Arora, J. S., 2004. " Survey of multiobjective optimization methods for engineering." In: Struct. Multidisc. Optimal, 26:369\{395\}

[6] Yang, X. S., 2010a. Engineering Optimization: An Introduction with Metaheuristic Applications, John Wiley and Sons, USA.

[7] Yang, X. S. and Gandomi, A. H., 2012. "Bat algorithm: a novel approach for global engineering optimization." Engineering Computations, 29(5):464\{483\}

[8] Schafier, J. D., 1985. "Multiple objective optimization with vector evaluated geneticalgorithms." in: Proc. 1st Int. Conf. Genetic Algorithms, pp. 93\{100\}

[9] Deb, K., Pratap, A., and Moitra, S., 2000. "Mechanical component design for multiple objectives using elitist nondominated sorting GA", in: Proceedings of the ParallelProblem Solving from Nature VI Conference, Paris, 16-20 Sept 2000, pp. 859-868.

[10] Babu, B. V. and Gujarathi, A. M., 2007. "Multi-objective differential evolution (MODE) for optimization of supply chain planning and management", in: IEEE Congress on Evolutionary Computation (CEC 2007), pp. 2732-2739.

[11] Xue, F., 2004.” Multi-objective differential evolution: theory and applications", PhD the-sis, Rensselaer Polytechnic Institute.

[12] Robific, T. and Filipific, B., 2005. "DEMO: differential evolution for multi-objective optimization.” in: EMO 2005 (eds. C. A. CoelloCoello), LNCS 3410:520\{533\}

[13] Pham, D. T. and Ghanbarzadeh, A., 2007." MultiObjective Optimization using the Bees Algorithm." in: 3rd International Virtual Conference on Intelligent Production Machines and Systems (IPROMS 2007), Whittles, Dunbeath, Scotland.

[14] Deb, K., Pratap, A., Agarwal, S., Mayarivan, T., 2002." A fast and elististmul-tiobjective algorithm: NSGA-II", IEEE Trans. Evol. Computation, 6(2):182-197.

[15] Douglas Rodrigues, Xin-She Yang, André Nunes de Souza and João Paulo Papa," Binary Flower Pollination Algorithm and Its Application to Feature Selection", Recent Advances in Swarm Intelligence and Evolutionary

Computation, Studies in Computational Intelligence 585, DOI 10.1007/978-3-319-13826-8_5

[16] José A. Regalado, Emilio Barocio E, Erick Cuevas , "Optimal Power Flow Solution Using Modified Flower Pollination Algorithm" Conference Paper - November 2015 ,978-1-4673-7121-6/15/\$31.00 @ 2015 IEEE

[17] Osama Abdel-Raouf, Mohamed Abdel-Baset and Ibrahim El-henawy," An Improved Flower Pollination Algorithm with Chaos", in: I.J. Education and Management Engineering, 2014, 2, 1-8. 
[18] Osama Abdel-Raouf, Ibrahim El-henawy, Mohamed Abdel-Baset," A Novel Hybrid Flower Pollination Algorithm with Chaotic Harmony Search for Solving Sudoku Puzzles", in: I.J. Modern Education and Computer Science, 2014, 3, 38-44.

[19] Mohamed Abdel-Baset, Ibrahim M. Hezam," An Effective Hybrid Flower Pollination and Genetic Algorithm for Constrained Optimization Problems", in: Adv. Eng. Tec. Appl. 4, No. 2, 27-34 (2015).

[20] O. Abdel-Raouf, M. Abdel-Baset, I. El-henawy,” A New Hybrid Flower Pollination Algorithm for Solving Constrained Global Optimization Problems", in: International Journal of Applied Operational Research Vol. 4, No. 2, pp. 1-13, Spring 2014.

[21] Dwaipayan Chakraborty, Sankhadip Saha, Oindrilla Dutta," DE-FP A: A Hybrid Differential EvolutionFlowerPollination Algorithm for Function Minimization", 978-1-4799-5958-7114/\$31.00 102014 IEEE.

[22] R.Jensi, G.Wiselin Jiji," HYBRID DATA CLUSTERING APPROACH USING K-MEANS AND FLOWER POLLINATION ALGORITHM", in: Advanced Computational Intelligence: An International Journal (ACII), Vol.2, No.2, April 2015.

[23] Hari Mohan Dubey, ManjareePandit, B.K. Panigrahi," Hybrid flower pollination algorithm with time-varying fuzzy selection mechanism for wind integrated multi-objective dynamic economicdispatch", 0960-1481/@ 2015 Elsevier Ltd. [24] NavdeepKaurJohal, Samandeep Singh, Harish Kundra," A hybrid FPAB/BBO Algorithm for Satellite ImageClassification", in: International Journal of Computer Applications (0975 - 8887) Volume 6- No.5, September 2010. [25] Emad Nabil," A Modified Flower Pollination Algorithm for Global Optimization", Article in Expert Systems with Applications - April 2016.

[26] Mohamed Abdel-Baset, Ibrahim Hezam," A Hybrid Flower Pollination Algorithm for Engineering Optimization Problems", in: international Journal of Computer Applications (0975 - 8887) Volume 140 - No.12, April 2016.

[27] Ibrahim M. Hezam, Mohamed Abdel-Baset, Bayoumi M. Hassan," A Hybrid Flower Pollination Algorithm with Tabu Search for Unconstrained Optimization Problems", Inf. Sci. Lett. 5, No. 1, 29-34 (2016).

[28] GaganpreetKaur, Dheerendra Singh, ManjinderKaur," Robust and Efficient 'RGB' based Fractal Image Compression: Flower Pollination based Optimization", in: International Journal of Computer Applications (0975 - 8887) Volume 78 - No.10, September 2013.

[29] MarwaSharawi, E. Emary, ImaneAlySaroit, Hesham ElMahdy," Flower Pollination Optimization Algorithm forWireless Sensor Network Lifetime GlobalOptimization", in: International Journal of Soft Computing and Engineering (IJSCE)ISSN: 2231-2307, Volume-4, Issue-3, July 2014.

[30] E. Emary, Hossam M. Zawbaa, Aboul Ella Hassanien, Mohamed F. Tolba and Vaclav Snasel," Retinal vessel segmentation based on flower pollination search algorithm". [31] MeriemBensouyad," A discrete flower pollination algorithm for graph coloring problem", Cybernetics (CYBCONF), 2015 IEEE 2nd International Conference on, Page(s):151 - 155,Print ISBN: 978-1-4799-8320-9.

[32] Wang, Rui; Zhou, Yongquan; Zhou, Yuxiang; Bao, Zongfan," Local Greedy Flower Pollination Algorithm for Solving Planar Graph Coloring Problem", in: Journal of Computational and Theoretical Nanoscience, Volume 12, Number 11, November 2015, pp. 4087-4096(10).
[33] Khalil AL-Wagih," An Improved Flower Pollination Algorithm for Solving Integer Programming Problems", in: Appl. Math. Inf. Sci. Lett. 3, No. 1, 31-37 (2015).

[34] Ibrahim El-henawy, Mahmoud Ismail," An Improved Chaotic Flower Pollination Algorithm for Solving LargeInteger Programming Problems",in: International Journal of Digital Content Technology and its Applications(JDCTA)Volume8, Number3, June 2014.

[35] Mohamed Abdel-Baset, Ibrahim M. Hezam," An Improved Flower Pollination Algorithm for Ratios Optimization Problems", in: Appl. Math. Inf. Sci. Lett. 3, No. 2, 83-91 (2015).

[36] RuiWang and Yongquan Zhou," Flower Pollination Algorithm with Dimension by Dimension Improvement", in: Hindawi Publishing Corporation Mathematical Problems in EngineeringVolume 2014, Article ID 481791, 9 pages.

[37] yongquan Zhou, Rui Wang," An Improved Flower Pollination Algorithm for Optimal UnmannedUndersea Vehicle Path Planning Problem", Article in International Journal of Pattern Recognition and Artificial Intelligence January 2016.

[38] Alberto Ochoa, Saúl González, Lourdes Margain, Teresa Padilla, Oscar Castillo, Patricia Melín," Implementing Flower Multi-objective Algorithm for selection of University academiccredits", August 2015.

[39] S. Sakthivel, P. Manopriya, S. Venus, S. Ranjitha and R. Subhashini," Optimal Reactive Power Dispatch Problem Solved By Using Flower Pollination Algorithm", in: International Journal of Applied Engineering Research ISSN 0973-4562 Volume 11, Number 6 (2016) pp 4387-4391.

[40] A. Sudheer K umarDr.A.V. 'Giridhar,' A New Meta Heuristic Algorithm Based ShuntCapacitive Compensation for Power LossReduction on Radial Distribution System", in: International Journal of Engineering Research \& Technology (IJERT) ISSN: 2278-0181Vol. 3 Issue 10, October- 2014.

[41] C. Shilaja, K. Ravi," Optimal Line Flow in Conventional Power System using Euclidean Affine Flower Pollination Algorithm", in: international journal of renewable energy research C.Shilaja and K.Ravi, Vol.6, No.1, 2016.

[42] PrernaSaxena and Ashwin Kothari," Linear antenna array optimization using flower pollination algorithm", Saxena and Kothari SpringerPlus (2016) 5:306.

[43] Mohammad Musofa Mulya Pambudy, Sasongko Pramono Hadi, Husni Rois Ali," Flower Pollination Algorithm for Optimal Control inMulti-Machine System with GUPFC", 2014 6th in: International Conference on Information Technology and Electrical Engineering (ICITEE), Yogyakarta, Indonesia.

[44] V. S. S. S. Chakravarthy Vedula,S. R. Chowdary Paladuga, and M. Rao Prithvi," Synthesis of Circular Array Antenna for Sidelobe Level and Aperture Size Control Using Flower Pollination Algorithm", in: Hindawi Publishing Corporation International Journal of Antennas and Propagation Volume 2015, Article ID 819712, 9 pages.

[45] Priyanka Das and Manoj Kumar ," Improvement of Voltage Profile using Shunt Capacitor in Radial Distribution System by Flower Pollination Algorithm and Sensitivity Analysis", in: Journal of Basic and Applied Engineering Research p-ISSN: 2350-0077; e-ISSN: 2350-0255; Volume 2, Number 18; July-September, 2015, pp. 1555-1559.

[46] Priyanka Das and Manoj Kumar," Voltage Profile Improvement using Distributed Generation in 33-bus and 69bus RDS by Flower Pollination Algorithm and Sensitivity Factor", in: Advanced Research in Electrical and Electronic Engineering p-ISSN: 2349-5804; e-ISSN: 2349-5812 Volume 2, Issue 14 October-December, 2015, pp. 37-41. 
[47] A.Y. Abdelaziz , E.S. Ali, S.M. AbdElazim ," Optimal sizing and locations of capacitors in radial distribution systems via flower pollination optimization algorithmand power loss index", in: Engineering Science and Technology, an International Journal 19 (2016) 610-618.

[48] Dinakara Prasad Reddya,, V.C. Veera Reddy, T. Gowri Manohara," Application of flower pollination algorithm for optimal placement and sizing of distributed generation in Distribution systems", in: Journal of Electrical Systems and Information Technology ARTICLE IN PRESS

[49] Eyad S. Oda , Abdelazeem A. Abdelsalam, Mohamed N. Abdel-Wahab ,Magdi M. El-Saadawi ," Distributed generations planning using flowerpollination algorithm for enhancing distributionsystem voltage stability", in: Ain Shams Engineering Journal (2015) ARTICLE IN PRESS .

[50] D. Lakshmi, A. Peer Fathima, Ranganath Muthu," A Novel Flower Pollination Algorithm to Solve Load Frequency Control for aHydro-Thermal Deregulated Power System", Circuits and Systems, 2016, 7, 166-178 Published Online April 2016 in SciRes.

[51] A.Y. Abdelaziz , E.S. Ali , S.M. AbdElazim ," Flower pollination algorithm to solve combined economic and emission dispatch problems", in: Engineering Science and Technology, an International Journal ARTICLE IN PRESS

[52] R. Prathiba, M. Balasingh Moses, S. Sakthivel," Flower Pollination Algorithm Applied forDifferent Economic Load Dispatch Problems", in: International Journal of Engineering and Technology (IJET) ISSN : 0975-4024 Vol 6 No 2 AprMay 2014.

[53] Hari Mohan Dubey, B.K. Panigrahi, and ManjareePandit," Improved Flower Pollination Algorithm for Short Term Hydrothermal Scheduling", (c) Springer International Publishing Switzerland 2015B.K. Panigrahi et al. (Eds.): SEMCCO 2014, LNCS 8947, pp. 721-737, 2015.

[54] Udjianna S. Pasaribu, Fathimah al-Ma'shumah, DonyPermana," Estimation of the Transition Matrix in Markov Chain Model of Customer Lifetime Value Using Flower Pollination Algorithm", in: Applied Mathematical Sciences, Vol. 9, 2015, no. 69, 3409 - 3418.

[55]Sinan Melih Nigdeli, Gebrail Bekdaş, Xin-She Yang," Application of the Flower Pollination Algorithm in Structural Engineering", Volume 7 of the series Modeling and Optimization in Science and Technologies pp 25-42. 